Revue de droit comparé du travail et de la sécurité sociale

2| 2018

Harcèlement moral au travail en jurisprudence

comparée

\title{
Approche comparative de la jurisprudence relative au harcèlement moral au travail
}

\section{Allison Fiorentino}

\section{(2) OpenEdition}

\section{Journals}

Édition électronique

URL : https://journals.openedition.org/rdctss/1942

DOI : $10.4000 /$ rdctss. 1942

ISSN : 2262-9815

Éditeur

Centre de droit comparé du travail et de la sécurité sociale

Édition imprimée

Date de publication : 1 juin 2018

Pagination : 6-21

ISSN : $2117-4350$

Référence électronique

Allison Fiorentino, « Approche comparative de la jurisprudence relative au harcèlement moral au travail », Revue de droit comparé du travail et de la sécurité sociale [En ligne], 2 | 2018, mis en ligne le 01 novembre 2021, consulté le 12 novembre 2021. URL : http://journals.openedition.org/rdctss/1942 ; DOI : https://doi.org/10.4000/rdctss. 1942

\section{(c) (i) $\ominus$}

Revue de droit comparé du travail et de la sécurité sociale est mise à disposition selon les termes de la Licence Creative Commons Attribution - Pas d'Utilisation Commerciale - Pas de Modification 4.0 International. 


\section{APPROCHE COMPARATIVE DE LA JURISPRUDENCE RELATIVE AU HARCELLEMENT MORAL AU TRAVAIL}
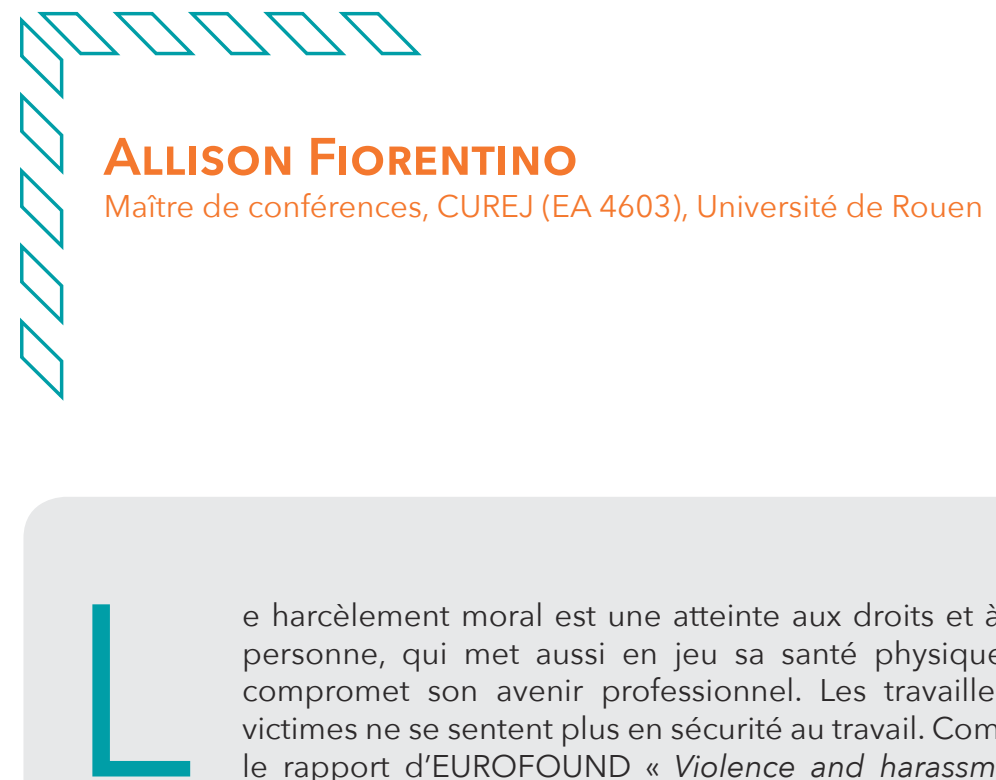

e harcèlement moral est une atteinte aux droits et à la dignité de la personne, qui met aussi en jeu sa santé physique et mentale ou compromet son avenir professionnel. Les travailleurs qui en sont victimes ne se sentent plus en sécurité au travail. Comme en témoigne le rapport d'EUROFOUND "Violence and harassment in European workplaces: Extent, impacts and policie ${ }^{1}$, les situations de harcèlement engendrent un taux d'absentéisme élevé jusqu'à une incapacité de travailler des personnes concernées.

Cette étude présente l'ampleur de la violence et du harcèlement au travail dans les 28 pays de I'Union Européenne et en Norvège. Elle repose sur des enquêtes nationales menées entre 2009 et 2013, ainsi que sur les résultats de la cinquième enquête européenne sur les conditions de travail d'EUROFOUND². Elle analyse les liens entre les conditions de travail et le harcèlement au travail, indique l'impact sur les travailleurs et les entreprises et compare les politiques élaborées par les gouvernements et les partenaires sociaux pour résoudre ce problème.

1 M. Giaconne, D. Di Nunzio, A. Fromm, O. Vargas, Violence and harassment in European workplaces: Extent, impacts and policies, European Foundation for the Improvement of Living and Working Conditions, 2015, https://www.eurofound.europa.eu/observatories/eurwork/comparative-information/ violence-and-harassment-in-european-workplaces-extent-impacts-and-policies

2 EUROFOUND, Fifth European Working Conditions Survey, Publications Office of the European Union, 2012,

https://www.eurofound.europa.eu/sites/default/files/ef publication/field ef document/ef1182en.pdf 
Les données recueillies montrent des tendances divergentes au fil du temps: la violence physique est en baisse, mais les autres formes de harcèlement non seulement ne régressent pas, mais augmentent. Dans l'ensemble, 14 \% des travailleurs en 2010 ont déclaré avoir été soumis à au moins une forme de harcèlement. En outre, les auteurs de ces actes se diversifient puisqu'on note une progression de la violence psychologique de la part de tiers. Cela résulte de la modification des conditions de travail qui exposent davantage de salariés au contact d'intervenants extérieurs (clients, salariés d'autres entreprises, formateurs, tertiarisation de l'économie, etc.). Les conditions de travail ont également évolué en raison d'une plus grande intensité de travail, de plus importantes exigences psychologiques et physiques, d'une insécurité de l'emploi croissante, de conflits en milieu de travail et de mauvaises pratiques managériales.

En outre, les résultats indiquent que les femmes déclarent généralement avoir subi plus de violence et de harcèlement que les hommes. Certains secteurs d'activité sont également plus exposés que d'autres. Il en est ainsi du secteur médico-social, des transports, de l'hôtellerie et de la restauration. Le rapport relève aussi que, dans de nombreux pays, les travailleurs intérimaires et les apprentis sont plus susceptibles d'en faire l'expérience que les travailleurs embauchés en contrat à durée indéterminée.

L'ampleur de l'exposition au harcèlement moral au travail varie à travers l'Europe. Les travailleurs de pays du sud de l'Europe sont ainsi moins susceptibles de déclarer subir un harcèlement que ceux situés au nord. Depuis 2000, ces derniers, qui connaissent des proportions relativement élevées de travailleurs déclarant avoir été victimes au moins une fois dans la vie professionnelle de violence et de harcèlement ${ }^{3}$, ont élaboré diverses politiques émanant de la loi ou de normes rédigées par les autorités chargées de l'environnement de travail pour les combattre. Les normes juridiques adoptées pour lutter contre le harcèlement et leur nature varient en Europe, reflétant à la fois les particularités culturelles et résultant des différents systèmes de droit du travail. La tâche du comparatiste est mise à l'épreuve des grandes divergences d'approches entre les différents systèmes juridiques tant le harcèlement moral ne fait pas l'objet d'une définition unique ni même d'une terminologie unifiée. Un travail de précision de la notion de harcèlement moral au travail à l'aide d'une approche comparée (I) est un préalable avant d'approfondir l'étude du droit prétorien relatif au harcèlement qui mettra en lumière l'attitude du juge (II). Selon le pays et la culture juridique, celui-ci a usé des moyens juridiques à sa disposition afin d'améliorer la protection du salarié harcelé.

3 Sur cette question, voir L. Lerouge, Les pays de l'Europe du Nord face à la prévention des risques psychosociaux au travail : droit, politique de prévention, dialogue social (Belgique, Norvège, Pays-Bas, Suède), Synthèse des Journées d'études internationales organisées à Bordeaux les 3 et 4 mars 2011, COMPTRASEC, Université Bordeaux https://halshs.archives-ouvertes.fr/halshs-00705217/document 


\section{I - LE HARCÈLEMENT MORAL EN DROIT COMPARÉ : UNE NOTION PROTÉIFORME}

Loin d'être une notion aisée à cerner le harcèlement moral se caractérise d'abord par des divergences tenant à la terminologie utilisée et au contenu de cette notion (A). De telles différences reposent sur des facteurs culturels, géographiques et politiques (B)

\section{A - LE CONSTAT DE LA DIVERGENCE}

Dès les premiers instants, le travail du chercheur en droit comparé se heurte à la difficulté liée aux différentes conceptions du harcèlement moral au travail selon le système juridique étudié. II est ainsi nécessaire de s'attarder sur les différentes manières d'aborder le « harcèlement moral au travail » selon différents pays. L'étape initiale de ce travail est terminologique. Si en France la notion de harcèlement moral correspond à une catégorie juridique, en revanche, dans de nombreux systèmes étrangers, le chercheur qui souhaite connaître la réponse légale ou jurisprudentielle apportée au harcèlement est confronté à différentes dénominations. Pour ne donner qu'un bref aperçu de ce problème, on emploiera comme langue étrangère principalement l'anglais. Une traduction littérale du terme français "harcèlement moral " (moral harassment) ne sera pas comprise par une majorité de juristes étrangers et ne sera pas utilisée dans leur pays d'origine.

Le législateur, le juge et la doctrine se réfèrent, suivant le pays, à des dénominations différentes. Il est possible de recenser entre autres les terminologies de "bullying " (intimidation ou harcèlement) ${ }^{4}$, « emotional abuse » (abus émotionnel) ${ }^{5}$, "psychological harassment » (harcèlement psychologique) ${ }^{6}$, " mobbing » (comportement agressif en

4 S. Einarsen, "The Nature and Causes of Bullying at Work », International Journal of Manpower, vol. 20, n' 1/2, 1999, pp. 16-27 ; S. Harthill, «Workplace Bullying as an Occupational Safety and Health Matter: A Comparative Analysis », Hastings International and Comparative Law Review, vol. 34, 2011, pp. 253-302 ; K. Lippel, "The Law of Workplace Bullying: An International Overview », Comparative Labor Law \& Policy Journal, vol. 32, n 1, 2010, pp. 1-13; A. O'Connell, "Bullying in the Workplace: A Gap in Irish Legislation? », Employment \& Industrial Relations Law, vol. 23, n² 2, 2013, pp. 13-16 ; D. Yamada, "The Phenomenon of "Workplace Bullying" and the Need for Status-Blind Hostile Work Environment Protection », Georgetown Law Journal, vol. 88, 2000, pp. 475-536.

5 L. Keashly, "Emotional Abuse in the Workplace: Conceptual and empirical issues ", Journal of Emotional Abuse, 1998, pp. 85-117 ; P. Lutgen-Sandvik, "The communicative cycle of employee emotional abuse. Generation and regeneration of workplace mistreatment», Management Communication Quarterly, vol. 16, n² 4, 2003, pp. 471-501; S. Harvey, L. Keashly, "Emotional abuse: how the concept sheds light on the understanding of psychological harassment (in Quebec) ", Perspectives interdisciplinaires sur le travail et la santé [En ligne], n 7-3, 2005, http://pistes.revues.org/3193

6 R. Cox, "Psychological Harassment Legislation in Québec: The First Five Years ", International Comparative Labor Law and Policy Journal, vol. 32, n 1, 2010, pp. 55-89 ; C. Gonçalves Gouveia, "From Laissez-Faire to Fair Play: Workplace Violence \& Psychological Harassment », University of Toronto Faculty of Law Review, vol. 65, 2007, pp. 137-166. 
groupe) $)^{7}$ ou encore « Power harassment » (harcèlement moral au Japon) ${ }^{8}$. Plusieurs auteurs qui se sont déjà penchés sur cette notion soulignent l'ambiguïté générée par cette diversité. Les termes sont tantôt utilisés comme des synonymes, tantôt font spécifiquement référence à des comportements particuliers ${ }^{9}$. Certains chercheurs ont essayé de dégager des points communs. Ainsi le professeur Branch considérait en 2008 que chaque définition nationale se référait à des actions ou des propos inappropriés ou déraisonnables ${ }^{10}$ tels que ridiculiser le salarié en public, le surveiller constamment, remettre en cause ses compétences, le menacer ou encore répandre à son propos de fausses rumeurs ${ }^{11}$.

Toutefois ce consensus semble bien maigre au regard des divergences qui concernent la qualification juridique du harcèlement moral au travail. Par exemple, sa reconnaissance par le juge requiert-elle une répétition d'actes ou un seul acte suffit-il ? Pour être reconnu, le harcèlement moral au travail est-il seulement descendant ou peut-il être aussi ascendant ou latéral ? Le harcèlement moral au travail peut-il être qualifié en l'absence d'intention malveillante de son auteur?

Toutes ces interrogations laissent deviner la difficulté de conceptualiser le harcèlement qui semble par nature protéiforme. Une autre distinction liée à la fois à la terminologie et à la conception du harcèlement oppose les systèmes juridiques basés sur la Common Law et ceux d'inspiration romano-germanique. Il semble que ces premiers englobent la notion de harcèlement dans celle plus large de discrimination. Ainsi le harcèlement dans ces pays n'est qu'une forme de discrimination basée par exemple sur le sexe ou l'appartenance ethnique. Au demeurant le harcèlement est une notion souvent plus utilisée dans le contexte du harcèlement sexuel qui a fait l'objet de nombreuses campagnes de prévention ${ }^{12}$.

7 P. Fischninger, "Mobbing": The German Law of Bullying ", Comparative Labor Law \& Policy Journal, vol. 32, n 1, 2010, pp. 153-184 ; N. Davenport, R. Schartz, G. Elliott, Mobbing: Emotional Abuse in the American Workplace, Civil Society Publishing, 1999.

8 L. Lerouge, S. Naito, «Bullying and Law in Japan » in Lerouge L. (ed.), Psychosocial Risks in Labour and Social Security Law: a Comparative Legal Overview, Springer, Serie: Aligning Perspectives on Health, Safety and Well-Being, 2017, p. 243-252.

9 S. Branch, «You Say Tomatoe and I say Tomato: Can we Differentiate Between Workplace Bullying and Other Counterproductive Behaviours? ", International Journal of Organisational Behaviour, vol. 13, n 2, 2008, pp. 4-17, sp. p. 4 ; L. Crawshaw, « Workplace Bullying? Mobbing? Harassment? Distraction by a Thousand Definitions ", Consulting Psychology Journal: Practice and Research, 2009 , vol. 61, n³, pp. 263-267, sp. p. 264 ; D. Yamada, "The Phenomenon of "Workplace Bullying" and the Need for Status-Blind Hostile Work Environment Protection », op.cit., sp. p. 480.

10 S. Branch, «You Say Tomatoe and I say Tomato: Can we Differentiate Between Workplace Bullying and Other Counterproductive Behaviours? », op. cit., sp. p. 5 ; S. Branch, S. Ramsay, M. Barker, "Workplace bullying, mobbing and general harassment: a review ", International Journal of Management Reviews, vol. 15, 2013, pp. 280-299.

11 Le Professeur Branch s'appuie sur des travaux antérieurs, notamment E. Bassman, Abuse in the workplace, Westport, CT: Quorum Books, 1992 ; C. Rayner, H. Hoel, «A summary review of literature relating to workplace bullying », Journal of Community \& Applied Social Psychology, vol. 7, $n^{\circ} 3$, 1997, pp. 181-191 ; D. Zapf, S. Einarsen, «Bullying in the workplace: Recent trends in research and practice-an introduction », European Journal of Work and Organizational Psychology, vol. 10, $\mathrm{n}^{\circ} 4$, 2001, pp. 369-373.

12 K. Lippell, «The Law of Workplace Bullying: An International Overview», op. cit., p. 5. 
En revanche, le terme de «bullying » fait référence à cette forme de pression psychologique totalement détachée de la discrimination ${ }^{13}$ mais pas nécessairement prise en compte par le législateur. C'est le cas par exemple en Australie ou aux États-Unis. Toutefois certaines provinces anglo-saxonnes font la différence et la notion de "workplace harassment " est totalement distincte de celle de discrimination et fait l'objet d'une règlementation spécifique. Ainsi la législation de la province du Saskatchewan au Canada comporte deux définitions faisant nettement référence à ces deux formes de harcèlement.

" "Harcèlement" signifie, toute conduite, commentaire, attitude, action ou geste d'une personne:

(i) qui: (a) est basé sur la race, la croyance, la religion, la couleur, le sexe, l'orientation sexuelle, l'état matrimonial, la situation familiale, le handicap, la taille ou le poids, l'âge, la nationalité, l'ascendance ou le lieu d'origine; ou -(b) sous réserve des paragraphes (3) et (4) affecte négativement le bien-être psychologique ou physique des travailleurs et dont l'auteur sait ou devrait raisonnablement savoir que cela humilierait ou intimiderait les travailleurs et

(ii) qui constitue une menace pour la santé ou la sécurité du travailleur $\aleph^{14}$.

Même si des chercheurs évoquent une récurrence de certains critères ${ }^{15}$ dont :

- une grande diversité d'actes négatifs pouvant causer des dommages psychologiques,

- l'existence de comportements directs et indirects,

- l'exclusion du travailleur,

- le caractère répété des actes,

- leur durée dans le temps,

- la difficulté à se défendre,

La définition du harcèlement moral diffère suivant les États. Certains n'ont pas adopté de définition stricte laissant une certaine liberté au juge. C'est le cas, par exemple, du Royaume-Uni dont l'article $1^{\text {er }}$ du Protection from Harassment Act dispose : "Une personne ne doit pas poursuivre une conduite - a) qui équivaut à du harcèlement d'autrui, et b) qu'il sait ou devrait savoir être un harcèlement de l'autre ${ }^{16}$. De même, en Finlande, l'article 28 du Occupational Safety and Health Act dispose : «Si le harcèlement ou d'autres traitements inappropriés d'un salarié se produit au travail et provoque des risques ou des dangers pour la santé du salarié, l'employeur, après avoir pris connaissance de cette affaire, doit, par tous les moyens disponibles, prendre des mesures pour remédier à cette situation ${ }^{17}$. On ne peut qu'opposer cette imprécision à la formulation de l'article L 1152-1 du Code du travail français, en vertu duquel : " aucun salarié ne doit subir les agissements répétés de

13 C. Caponecchia, A. Wyatt, «Distinguishing between workplace bullying, harassment and violence: a risk management approach ", Journal of Occupational Health and Safety, Australia and New Zealand, vol. 25, nº 6, 2009, pp. 439-449.

14 Art. 3(1)(b) du Occupational Health and Safety (Harassment Prevention) Amendment Act, 2007, S.S. ch. 34 ; http://www.publications.gov.sk.ca/freelaw/documents/english/Chapters/2007/Chap-34.pdf

15 M. Varta-Väänänen, « Workplace Bullying and Harassment in the EU and Finland » in Workplace Bullying and Harassment, 2013 JILPT Seminar on Workplace Bullying and Harassment, op. cit., sp. p. 2.

16 Protection from Harassment Act 21st March 1997, https://www.legislation.gov.uk/ukpga/1997/40

17 Traduction en anglais: Occupational Safety and Health Act, $n^{\circ}$ 738/2002,

http://www.finlex.fi/fi/laki/kaannokset/2002/en20020738.pdf 
harcèlement moral qui ont pour objet ou pour effet une dégradation des conditions de travail susceptible de porter atteinte à ses droits et à sa dignité, d'altérer sa santé physique ou mentale ou de compromettre son avenir professionnel ».

\section{B - LES FACTEURS DE DIVERGENCE}

Les divergences précédemment constatées ne peuvent guère s'atténuer car elles s'expliquent d'abord par la diversité des sources d'inspiration du législateur ou du juge ou des zones géographiques. Ainsi, le Professeur Lippel ${ }^{18}$ fait une distinction entre deux zones principales d'influence : le droit anglo-saxon (États-Unis ${ }^{19}$, Royaume-Uni ${ }^{20}$ et Australie ${ }^{21}$ ) qui privilégie l'appellation de «workplace bullying » et les pays plutôt francophones ou latins qui ont été influencés par le travail de la psychiatre Marie-France Hirigoyen qui a conceptualisé le harcèlement moral au travail en $1998^{22}$. Dans ce dernier cas, la terminologie employée est soit " harcèlement moral » ("moral harassment ») comme en France, en Belgique ${ }^{23}$ ou dans les pays de langue espagnole ${ }^{24}$ soit « harcèlement psychologique » au Québec ${ }^{25}$. Une troisième zone $d^{\prime}$ influence englobe les pays scandinaves ${ }^{26}$ et l'Allemagne ${ }^{27}$ marqués par

18 K. Lippell, «The Law of Workplace Bullying: An International Overview», The Comparative Labour Law \& Policy Journal, vol. 32, n² 1, 2010, pp. 1-13, sp. p. 1.

19 D. Yamada, "The Phenomenon of 'Workplace Bullying' and the Need for Status-Blind Hostile Work Environment Protection », op.cit.

20 H. Hoel, «Workplace Bullying in United Kingdom », in Workplace Bullying and Harassment, JILPT Report n 12/2013 - JILPT Seminar on Workplace Bullying and Harassment, 2013, pp. 61-76, http://www.jil.go.jp/english/reports/documents/jilpt-reports/no.12.pdf

21 B. French, M. Boyle, O. Muurlink, "Workplace Bullying in Australia: The Fair Work Act and its Impact », New Zealand Journal of Human Resources Management, vol. 14, n² 2, 2014, pp. 69-81.

22 M.-F. Hirigoyen, Le harcèlement moral. La violence perverse au quotidien, Pocket, 1998, 252 p.

23 L. Lerouge, " Workplace Bullying and Harassment in France and Few Comparisons with Belgium: a Legal Perspective », in Workplace Bullying and Harassment, JILPT Report $n^{\circ}$ 12/2013, op. cit., pp. 39-59.

24 En espagnol, la traduction littérale de harcèlement moral est « acoso moral ». A. Jurado Segovia, Acoso moral en el trabajo: análisis jurídico-laboral, La Ley, 2008. On note d'ailleurs un intérêt certain des éditeurs espagnols pour le travail de Marie-France Hirigoyen. M.-F., Hirigoyen, El acoso moral: el maltrato psicológico en la vida cotidiana, Grupo Planeta (GBS), 1999 ; El acoso moral en el trabajo: distinguir lo verdadero de lo falso, Grupo Planeta (GBS), 2001 ; El acoso moral, Grupo Planeta Spain, 2013.

25 R. Cox, "Psychological harassment legislation in Québec: the first five years ", The Comparative Labour Law \& Policy Journal, vol. 32, n 1, 2010, pp. 55-89 ; S. Harvey, L. Keashly, "Emotional abuse: how the concept sheds light on the understanding of psychological harassment (in Quebec) ", op. cit. Le professeur Goncalves Gouveia soulignait d'ailleurs que l'ouvrage de Marie-France Hirigoyen (Le Harcèlement Moral : La Violence Perverse au Quotidien) avait été un best seller au Québec. C. Gonçalves Gouveia, «From Laissez-Faire to Fair Play: Workplace Violence \& Psychological Harassment $»$, op. cit., sp. note 60.

26 M. I. S. Guerrero, "The Development of Moral Harassment (or Mobbing) Law in Sweden and France as a Step Towards EU Legislation", Boston College International and Comparative Law Review, vol. $27, n^{\circ} 2,2004$, pp. 477-500.

27 P. Fischinger, "'Mobbing': The German Law of Bullying », Comparative Labor Law \& Policy Journal, op. cit. 
les recherches du professeur d'origine germanique Heinz Leymann ${ }^{28}$ qui emploie volontiers le terme de "mobbing». Enfin certains pays utilisent des notions qui leur sont propres. Ainsi, au Japon, il est fait spécifiquement référence au « power harassment ${ }^{29}$.

Ces différences s'expliquent également par le contexte de l'adoption des dispositions légales. Ainsi certains pays furent influencés par les travaux de chercheurs, pionniers en la matière, notamment Heinz Leymann, Christophe Dejours, et de praticiens comme le docteur Marie-France Hirigoyen ${ }^{30}$.

Il faut également mentionner l'existence d'autres textes antérieurs relatifs à la protection de la santé mentale du salarié qui constituèrent le point de départ, dans d'autres pays, de l'émergence d'une règlementation sur le harcèlement. À ce titre nous pouvons citer la directive 89/391/CEE du Conseil du 12 juin 1989 concernant la mise en œuvre des mesures visant à promouvoir l'amélioration de la sécurité et de la santé des travailleurs au travail ${ }^{31}$. Le texte communautaire eut une incidence non négligeable et jouera un rôle certain dans l'adoption de dispositions légales en France et en Belgique ${ }^{32}$ ou de codes de bonne conduite anti harcèlement en Espagne ${ }^{33}$.

Ce sont également les circonstances factuelles qui contraignent les autorités étatiques à adopter certaines mesures, comme en Corée du Sud. En 1997, lorsque la crise économique frappa ce pays, le sort des travailleurs se dégrada subitement. Non seulement les entreprises exigèrent des performances toujours plus élevées sous la menace d'un licenciement, mais elles utilisèrent aussi le harcèlement comme moyen de sélection lors des procédures de compression du personnel. En outre, l'atmosphère de travail était tellement délétère que certains travailleurs firent l'objet de persécution de la part de leurs collègues, notamment au moyen de calomnies répandues grâce aux médias sociaux, le but étant de pousser à la démission le concurrent dont on convoitait le poste ${ }^{34}$. Cette situation poussa le ministre du Travail à adopter, pour la première fois, en 1999, une règlementation.

28 H. Leymann, «The content and development of mobbing at work», European Journal of Work and Organizational Psychology, vol. 5, n² 2, 1996, pp. 165-184.

29 L. Lerouge, S. Naito, « Harcèlement, «Power Harassment» et droit au Japon » in Lerouge L. (dir), Les risques psychosociaux au travail en droit social : approche juridique comparée. France Europe - Canada - Japon, Dalloz, 2014, p. 211-221 ; S. NAITO, « Workplace Bullying in Japan », in Workplace Bullying and Harassment, JILPT Report n 12/2013, op. cit., pp. 113-133.

30 K. Lippell, "The Law of Workplace Bullying: An International Overview », op. cit., p. 6 ; H. Hoel, S. Einarsen, "The Swedish Ordinance Against Victimization at Work' A Critical Assessment", Comparative Labor Law \& Policy Journal, 2010, vol. 32, n 1, pp. 225-250.

31 S. Leka, A. Jain, S. lavicoli, C. Di Tecco, « An Evaluation of the Policy Context on Psychosocial Risks and Mental Health in the Workplace in the European Union: Achievements, Challenges, and the Future », BioMed Research International, 2015, https://www.ncbi.nlm.nih.gov/pmc/articles/PMC4628767/

32 L. Lerouge, "Workplace Bullying and Harassment in France and Few Comparisons with Belgium: a Legal Perspective " in Workplace Bullying and Harassment, JILPT Report n 12/2013, op. cit., sp. p. 39

33 M. Velazquez, "The Spanish Code of Practice on Work-Related Bullying: Reflections on European Law and its Impact on a National Strategy For Labor Inspectors", Comparative Labor Law \& Policy Journal, 2010, vol. 32, n 1, pp. 185-224.

34 S. Park, "Workplace Bullying and Harassment in South Korea», in Workplace Bullying and Harassment, JILPT Report n¹2/2013, op. cit., sp. p. 97. 


\section{II - LE RÔLE DE LA JURISPRUDENCE : APPROCHE COMPARATIVE EN DROIT FRANÇAIS, BRITANNIQUE, JAPONAIS, ESPAGNOL ET AMÉRICAIN}

Certaines juridictions ont fait preuve d'audace ou, à tout le moins, d'une volonté de protection au bénéfice des salariés. À ce titre nous pouvons citer les cours françaises, japonaises et, dans une moindre mesure britannique $(A)$. En revanche, les juges américains et espagnols sont peu enclins à user de leur pouvoir prétorien pour apporter davantage de garanties au salarié harcelé (B)

\section{A - L'AUDACE PRÉTORIENNE DES JUGES FRANÇAIS, BRITANNIQUES ET JAPONAIS}

Le droit français relatif au harcèlement moral est redevable au législateur tout autant qu'au juge ${ }^{35}$. Si c'est la loi du 17 janvier $2002^{36}$ qui a introduit cette notion dans le Code du travail, la jurisprudence avait déjà eu l'occasion de se saisir de ce problème. Les juges du fond, sans recourir à la terminologie de harcèlement qualifiaient de « faute » l'attitude de l'employeur qui consiste à dévaloriser, humilier, ou pousser à la démission le salarié. II en va ainsi de l'employeur qui affirme devant témoin que la salariée n'est bonne à rien, qu'elle n'est pas indispensable et qui l'a fait sortir de son bureau en pleurs en la poursuivant de ses cris $^{37}$. En outre, et avant même la reconnaissance de l'obligation de sécurité de résultat à la charge de l'employeur, le juge français rendait les fautes de certains tiers imputables à l'employeur. Par exemple : lorsque le salarié a subi nombre d'actions déstabilisantes de la part du conjoint de l'employeur; ou encore lorsque l'employeur n'a rien fait pour faire cesser I'hostilité du personnel à laquelle se heurtait le salarié et a, au contraire, encouragé cette attitude ${ }^{38}$. Comme le notait Mme Béatrice Lapérou « le concept juridique de harcèlement moral émerge lentement des décisions juridiques $»^{39}$.

$\mathrm{Si}$ cette jurisprudence était novatrice, elle n'avait rien de révolutionnaire car les juridictions du fond n'avaient nul besoin de recourir à un fondement fantaisiste pour justifier leur décision. L'obligation protéiforme de bonne foi - notamment dans l'exécution du contrat de travail - était toute désignée pour légitimer cette construction prétorienne.

L'intervention du législateur en 2002 n'a pas pour autant tari l'inventivité prétorienne lorsqu'il s'agit de protéger le salarié harcelé. L'obligation de sécurité de résultat, opportunément découverte en $2002^{40}$, eut une incidence non négligeable en matière de harcèlement car elle conduisit le juge à admettre en 2010 la responsabilité de l'employeur

35 Voir également l'article de Loïc Lerouge publié dans le numéro.

36 Loi n ${ }^{\circ} 2002-73$ du 17 janvier 2002 de modernisation sociale.

37 CA Toulouse 31 mars 2000, $4^{e}$ ch. soc., SARL P. c/ R, RJS 4/01, n 404. Voir également CA Toulouse 18 mai 2000, 4e ch. soc. SNC Caisse de prévoyance et de retraite de la SNCF c/ Gelardo et CA Nancy 13 novembre 2000, ch. soc., Christophe c/ SA Vilroc, RJS 2/01, n 163.

38 CA Poitiers 30 mai 2000, ch. soc., Chaigneau c/ Morisset et CA Bordeaux 3 mars 2000, ch. soc. B, SA Marbot et Cie c/ Durepaire ; RJS 12/00 n 1218.

39 B. Laperou-Scheneider, "La notion de harcèlement moral dans les relations de travail », RJS 6/00, pp. 423-433.

40 Cass. soc. 28 février 2002, Bull., V, n 81, p. 84 ; Babin M., Pichon N., "Obligation de sécurité et faute inexcusable de l'employeur », Dr. soc. sept.-oct. 2002, p. 828 ; Lyon-Caen A., « Une révolution dans le droit des accidents du travail », Dr. soc., avr. 2002, p. 445. 
lorsqu'un salarié est harcelé « quand bien même il aurait pris des mesures en vue de faire cesser ces agissements ${ }^{41}$. Si cette jurisprudence a évolué plus récemment, le juge fait toujours preuve de sévérité envers l'employeur puisqu'il est exonéré de sa responsabilité seulement s'il a pris toutes les mesures immédiates propres à faire cesser le harcèlement moral et qu'il l'a fait cesser effectivement. II importe également qu'il ait pris toutes les mesures de prévention visées aux articles L. 4121-1 et L. 4121-2 du Code du travail, et notamment qu'il ait préalablement mis en œuvre des actions d'information et de formation propres à prévenir la survenance de faits de harcèlement moral ${ }^{42}$.

Le juge français n'est pas le seul à avoir suppléé aux carences légales. Le juge britannique peut également être cité en exemple. De manière intéressante, il est à noter que les magistrats britanniques poursuivirent une évolution juridique que le législateur avait abandonnée, peut-être par manque de courage politique. Ainsi l'arrivée au pouvoir du New Labour Party et de son charismatique leader Tony Blair suscita une vague d'espoir auprès de syndicats qui déploraient la dégradation des conditions de travail et avaient initié une campagne pour la dignité au travail ${ }^{43}$. Une proposition de loi intitulée "Dignité au travail » fut déposée peu de temps avant la victoire du parti travailliste. Toutefois, et dépit des espoirs des syndicats, ce texte ne fut jamais adopté ${ }^{44}$.

En revanche, le législateur adopta un texte d'une portée plus générale : le Protection from Harassment Act en $1997^{45}$. Cette loi fut promulguée pour permettre une réparation pénale et civile à la victime d'un harcèlement. Les débats parlementaires établissent que la forme de discrimination envisagée était celle classique d'un individu obsédé qui utilise toute sorte de moyen pour poursuivre de ses attentions sa victime. Néanmoins, plusieurs membres du Parlement soulignèrent que la terminologie usitée par le texte légal avait un champ d'application si large qu'il était susceptible de s'appliquer en droit du travail. Les tribunaux leur donnèrent raison rapidement non seulement en reconnaissant la responsabilité du harceleur sur ce fondement mais également, de manière plus intéressante, en recevant favorablement les demandes en réparation contre l'employeur au titre de sa responsabilité de commettant (vicarious liability) alors même que cette possibilité n'était pas envisagée par la loi. La première décision en la matière remonte à 2005.

41 Cass. soc. 3 février 2010, nº 08-40.144; J. MOULY, «Obligation de sécurité de l'employeur et harcèlement «horizontal» : vers une obligation de résultat absolue ? ", JCP G 2010, n 12, 321 ; S. Tournaux, "La vigueur retrouvée de l'obligation de sécurité de résultat», Lexbase Hebdo - Edition Sociale 18/02/2010, $n^{\circ} 383$.

42 Cass. soc., 1 er juin 2016, n 14-19.702 ; voir notamment B. Geniaut, "Le harcèlement moral dans la jurisprudence sur l'obligation de sécurité », RDT 2016, n 11, pp. 709-711; G. Loiseau, "Le renouveau de l'obligation de sécurité », JCP S 2016, n²4, pp. 27-30; C. RADE, "Feue la responsabilité de plein droit de l'employeur en matière de harcèlement : le mieux, ennemi du bien », Lexbase Hebdo - Edition Sociale, 16/06/2016, nº 659.

43 S. Harthill, «Bullying in the Workplace: Lessons from the United Kingdom », Minnesota Journal of International Law, vol. 17, 2008, pp. 247-302, sp. p. 252.

44 La proposition de loi sur la dignité au travail (HL Bill 31.53/1) fut déposée par Lord Monkswell à la Chambre des Lords en décembre 1996 mais fut bloquée par le gouvernement conservateur de John Major en février 1997. Elle fut réintroduite sans succès devant la Chambre des lords en décembre 2001.

https://publications.parliament.uk/pa/ld200102/ldbills/031/2002031.htm

45 Op. cit. 
Dans l'arrêt Majrowski"4, la Court of Appeal a jugé que la Sécurité sociale britannique était responsable du fait d'autrui en raison du harcèlement subi par William Majrowski et qui était le fait de son chef de service. L'employeur fut condamné à verser des dommages et intérêts alors même qu'il n'avait pas pris part aux persécutions subies par le salarié. La jurisprudence ultérieure confirma la règle de l'arrêt Majrowski. Une attention particulière peut être portée à un jugement d'août 2006, qui a octroyé à une salariée requérante 800000 f de dommages-intérêts ${ }^{47}$. La demanderesse n'a pas jugé bon de poursuivre les auteurs des actes mais a engagé la responsabilité de son employeur. La salariée avait été embauchée par la Deutsche-Bank Group Services comme secrétaire adjointe. Dès son recrutement, elle fut soumise à une campagne de harcèlement de la part de quatre de ses collègues. Les actes d'intimidation consistaient en une multitude de mesquineries destinées à la dévaloriser : déplacer ses papiers, éclater de rire quand elle passa devant eux, faire des remarques grossières et obscènes, cacher son courrier, la retirer des listes de diffusion de documents, ou encore augmenter sa charge de travail de manière déraisonnable pour la mettre en situation d'échec.

Produisant les attestations d'autres salariés, la requérante emporta la conviction des juges qui lui donnèrent gain de cause en lui accordant cette somme très importante. Dans cette même décision, le juge apporta des clarifications à la notion de harcèlement. La cour considéra que le harcèlement : a) se produit au moins à deux reprises, (b) cible le demandeur, (c) est objectivement destiné à causer le mal être de la victime, et d) est objectivement oppressant et déraisonnable.

Cette jurisprudence fut maintenue et les indemnités octroyées sur ce fondement peuvent être ruineuses pour les employeurs. Un record a d'ailleurs été battu lorsqu'une avocate obtint 1,3 million $f$ pour avoir été victime de harcèlement ${ }^{48}$. Non seulement les juridictions britanniques ont retenu la responsabilité de l'employeur commettant avec une sévérité qui n'a rien à envier à celle dont ont fait preuve les juges français lorsqu'ils se fondèrent sur l'obligation de sécurité, mais une autre comparaison peut faite entre ces deux jurisprudences nationales.

De même que le juge français a usé de l'obligation de bonne foi, la jurisprudence britannique s'est appuyée sur le droit commun des obligations pour reconnaître, au profit du salarié, une protection contre le harceleur. Tout d'abord, dans les années 1990 et indépendamment de toute disposition légale, les magistrats ont admis que le harcèlement puisse constituer un délit civil justiciable ${ }^{49}$. En droit du travail, ce délit civil fut reconnu dans un contexte particulier, pour la première fois au milieu des années 1990 dans la décision Walker ${ }^{50}$. Sans faire référence au harcèlement moral, le juge admit la responsabilité de

46 Majrowski v. Guy's \& St. Thomas's NHS Trust, [2005] EWCA Civ 251.

47 Green v. DB Group Services (UK) Ltd, [2006] EWHC 1898 (Q.B.).

48 S. Harthill, «Bullying in the Workplace: Lessons from the United Kingdom», op. cit., p. 284, note 198.

49 Burris v. Azadani, (1995) 1 WLR 1372. Cette décision concernait le harcèlement dont était victime une femme et son enfant. Le contexte n'était pas le droit du travail mais la reconnaissance de ce nouveau délit civil était susceptible d'applications en ce domaine.

50 Arrêt Walker v Northumberland County Council de la Queen's Bench Division ; [1995] 1 All ER 737 ; [1995] ICR 702 ; [1995] IRLR 35 ; V. R. Walden, « Employer Responsibility for the Psychosocial and Psychiatric Well-Being of Employees in the Workplace: A View from the United Kingdom » in L. Lerouge (ed.), Psychosocial Risks in Labour and Social Security Law: a Comparative Legal Overview, Springer, Serie: Aligning Perspectives on Health, Safety and Well-Being, 2017, pp. 79-104. 
l'employeur harceleur lorsque le salarié est victime d'un grave préjudice psychologique. Plus précisément le juge considéra que cela pouvait caractériser le délit civil de negligence ${ }^{51}$ qui impose, au titre de ses conditions d'exercice, la preuve d'un devoir spécifique de diligence dont le défendeur serait débiteur (et qui, par hypothèse, n'aurait pas été respecté). En l'espèce ce devoir de vigilance que l'employeur méconnait est le devoir de tout employeur de fournir à ses salariés un environnement de travail convenable ${ }^{52}$.

Dans l'arrêt Walker, le demandeur avait souffert d'une dépression nerveuse en 1986 et avait bénéficié de quatre mois de congé. Cependant son employeur avait refusé de lui apporter une aide supplémentaire en le déchargeant d'une partie de son travail alors que cette mesure était envisageable. Au contraire, la charge de travail s'alourdit, résultant en une seconde dépression aux effets très sévères qui aboutit au licenciement du salarié. Ce dernier demanda et obtint des dommages et intérêts de son employeur. Le juge considéra que l'employeur était responsable pour la dépression nerveuse induite par le stress au travail. Le tribunal souligna que dans une telle situation le demandeur, pour obtenir gain de cause, devait établir que les mesures requises de l'employeur étaient raisonnables, que les risques encourus par le salarié étaient importants et prévisibles. Ces conditions étaient en l'espèce remplies.

Cette jurisprudence, pour utile qu'elle soit au salarié, a malheureusement un champ d'application assez restreint car elle ne concerne que les préjudices psychologiques résultant du stress au travail et non pas le harcèlement moral en tant que tel ${ }^{53}$.

Un autre fondement a également été admis par le juge : la loi Employment Rights Act $1996^{54}$. Cette loi dispose que les salariés ne peuvent être injustement licenciés. En outre, la loi inclut dans la notion de licenciement, celle de "congédiement déguisé "55 qui fait référence à des situations dans lesquelles un salarié est forcé de quitter son travail contre sa volonté en raison de la conduite de son employeur (un parallèle peut être fait avec la notion française de prise d'acte). Au titre des fautes de l'employeur justifiant une rupture à ses torts, on peut citer le manquement au devoir de confiance réciproque. Ce devoir implicite, présent dans tout contrat de travail a été décrit comme « occupant une place centrale en droit du contrat de travail ${ }^{56}$ », « indubitablement le plus puissant des engins de mouvement du droit moderne des contrats de travai ${ }^{57}$ » ou encore « la pierre angulaire de

51 Le délit civil de négligence est caractérisé en présence d'une omission fautive à exercer les soins appropriés dans des circonstances précises. Le droit positif en la matière résulte de l'arrêt déjà ancien Donoghue v. Stevenson [1932] AC 562. Ce chef de demande nécessite plusieurs conditions notamment la preuve selon laquelle le défendeur était tenu d'un devoir spécifique de diligence.

52 Wilsons and Clyde Coal Co Ltd v English [1938] AC 57.

53 D. Leclie, J. MC Williams, "Stress at work. The implications for employers », Employment Law Bulletin 2001, n 44, pp. 6-8 ; V. Craig, "Civil liability for health and safety », Employment Law Bulletin 2002, $n^{\circ} 48$, pp. 5-8 ; B. Barrett, «Employers'liability for stress at the work place : neither tort nor breach of contract », Industrial Law Journal 2004, vol. 33, pp. 343-349.

54 Employment Rights Act 1996, https://www.legislation.gov.uk/ukpga/1996/18/contents

55 La doctrine désigne cette démission requalifiée de licenciement sous le terme: " constructive dismissal ». Employment rights Act 1996, art. 95.

56 D. Brodie, "Mutual Trust and the Values of the Employment Contract », Industrial Law Journal 2001, vol. 30, n 1, pp. 84-100, sp. p. 86

57 M. Freedland, The Personal Employment Contract, Oxford University Press, 2003, p. 166. 
la construction juridique du contrat de travail ${ }^{58}$ ». L'importante doctrine à ce sujet ${ }^{59}$ reflète l'intérêt prétorien pour le devoir de confiance réciproque qui impose à l'employeur d'agir avec une certaine loyauté.

La jurisprudence a utilisé ce devoir implicite pour sanctionner l'employeur harceleur lorsque son attitude conduit un salarié à quitter l'entreprise pour mettre fin à une situation intolérable ${ }^{60}$.

Indépendamment de l'intervention du législateur, le juge a su adapter le droit prétorien à l'évolution du monde du travail qui met davantage en lumière les effets dévastateurs du harcèlement.

Un autre exemple encore plus éclatant de la prise de conscience par le juge de la nécessité de lutter contre le harcèlement moral réside dans la jurisprudence japonaise ${ }^{61}$. Plus précisément, le droit japonais a adopté une théorie de la responsabilité délictuelle appelée harcèlement par le pouvoir (pawā harasumento ou pawahara), qui protège les employés contre les abus de ceux qui disposent de plus de pouvoir dans l'entreprise ${ }^{62}$. Cette question n'est pas nouvelle. Dès 1995, les autorités publiques de Tokyo avaient admis le caractère fondé des demandes d'indemnisation pour harcèlement ${ }^{63}$. Ce qui est, en revanche, plus récent c'est l'augmentation du nombre de plaintes pour ce motif. L'administration du travail de Tokyo notait que ce chiffre était passé de 3160 à 5960 de 2002 à $2008^{64}$. La crise économique qui a frappé le Japon n'a fait qu'amplifier ce phénomène. Les victimes de harcèlement, face à l'absence de disposition légale, se tournèrent vers le droit des obligations afin d'obtenir réparation. En la matière deux fondements étaient envisageables. Tout d'abord, l'article 709 du Code civil japonais qui impose à une personne qui a intentionnellement ou par négligence enfreint les droits d'autrui d'indemniser

58 H. Collins, Employment Law, Oxford University Press, 2003, p 104.

59 L. Barmes, «The Continuing Conceptual Crisis in the Common Law of the Contract of Employment », Modern Law Review 2004, Vol. 67, n 3, pp. 435-464; D. Brodie, "The Heart of the Matter: Mutual Trust and Confidence », Industrial Law Journal 1996, vol. 25, n², pp. 121-136; «Beyond Exchange: The New Contract of Employment », Industrial Law Journal 1998, vol. 27, n² 2, pp. 79-102; "A Fair Deal at Work», Oxford Journal of Legal Studies 1999, vol. 19, n 1, pp. 83-98; " Mutual Trust and Confidence: Catalysts, Constraints and Commonality », Industrial Law Journal 2008, vol. 37, $\mathrm{n}^{\circ} 4$, pp. 329-346 ; A. Brooks, « The Good and Considerate Employer: Developments in the Implied Duty of Mutual Trust and Confidence », University of Tasmania Law Review 2001, vol. 20, n 1, pp. 29-69; D. Cabrelli, «The Implied Duty of Mutual Trust and Confidence: An Emerging Overarching Principle?», Industrial Law Journal 2005, vol. 34, n 4, pp. 284-307; M. Freedland, "Constructing Fairness in Employment Contracts », Industrial Law Journal 2007, vol. 36, n 1, pp. 136-140 ; J. Lindsay, "The Implied Term of Trust and Confidence », Industrial Law Journal 2001, vol. 30, n 1, pp. 1-16.

60 Abbey National Plc v Robinson (EAT/743/99).

61 Voir également l'article du Professeur Kanta Owada publié dans le numéro.

62 P. Hsiao, "Power Harassment: The Tort of Workplace Bullying in Japan », Pacific Basin Law Journal, vol. 32, $n^{\circ}$ 2, 2015, pp.181-201 ; L. Lerouge, S. Naito, « Harcèlement, «Power Harassment» et droit au Japon » in Lerouge L.(dir), Les risques psychosociaux au travail en droit social : approche juridique comparée. France - Europe - Canada - Japon, op. cit. ; S. Naito, "Workplace Bullying in Japan ", in Workplace Bullying and Harassment, 2013 JILPT Seminar on Workplace Bullying and Harassment, op. cit., pp. 113-133.

63 P. Hsiao, « Power Harassment: The Tort of Workplace Bullying in Japan », op. cit., spéc. p. 183.

64 ld. 
les dommages qui en résultent ${ }^{65}$. Ce ne fut pas la base juridique choisie mais plutôt la seconde. En effet, les employeurs japonais sont tenus d'une obligation générale de veiller à ce que la santé mentale et physique d'un employé ne soit pas endommagée par l'accumulation excessive de stress qui accompagne l'exécution du travail ${ }^{66}$. La formulation est plus restrictive que l'obligation de sécurité française mais elle n'en impose pas moins aux employeurs d'être vigilants lorsque les salariés sont soumis à une charge de travail importante. Sur le fondement de cette obligation générale, les demandes d'indemnisation pour harcèlement ont prospéré au Japon.

Lesjuridictions japonaisesn'ont jamais défini le power harassment; mais la jurisprudence en la matière permet de mieux cerner cette notion. II semble que les magistrats soient enclins à donner gain de cause aux requérants salariés dans trois situations : le suicide, une gestion du personnel très stressante, et les conduites outrageantes. A titre d'exemple, en 2012, un tribunal de Saitama ${ }^{67}$ a accordé des dommages-intérêts aux parents d'une infirmière décédée et a condamné son collègue et son employeur en raison d'intimidations répétées qui l'ont conduite au suicide. Dans cette affaire, l'employeur, un hôpital, avait une règle obligeant les infirmières à suivre tout ordre donné par des infirmières supérieures, et l'infirmière en chef avait abusé de son autorité notamment :

- en contraignant sa subordonnée à travailler pour elle en lavant sa voiture et en prenant soin de son fils aîné,

- en forçant sa subordonnée à verser de l'argent pour avoir de bonnes relations avec ses supérieurs ;

- en tenant des propos très durs à la salariée, lui intimant à plusieurs reprises l'ordre de se taire et lui suggérant de mourir,

- en critiquant en public ses erreurs au travail.

Autre exemple : dans une décision de 2009, un tribunal de Tottori a accordé des dommages-intérêts à un salarié qui avait été victime d'une dépression causée par le stress et avait finalement quitté son emploi après que des gestionnaires l'aient réprimandé en public à plusieurs reprises ${ }^{68}$.

Au titre des conduites outrageantes générant une responsabilité du harceleur, voire de l'employeur s'il est complice, on peut retenir toutes les attitudes profondément humiliantes qui n'ont d'autres buts que détruire psychologiquement le salarié. Ainsi, un tribunal a accordé des dommages-intérêts aux requérants dont le patron avait installé un

65 Voir une traduction en anglais du code à l'adresse : http://www.moj.go.jp/content/000056024.pdf A l'image du juge français qui a usé de la théorie de l'abus de droit pour protéger le salarié contre le licenciement abusif, le juge japonais a également eu recours à cette théorie. K. Sono, Y. Fujioka, "The Role of the Abuse of Right Doctrine in Japan ", Louisiana Law Review, vol. 35, n 5, 1975, pp. 1037-1057. En droit du travail, cela a été utilisé pour sanctionner l'employeur qui procédait de manière trop brutale à un licenciement pour motif économique sans même tenter de reclasser le salarié. T. Suzuki, « Le pouvoir créateur du juge en matière de licenciement économique au Japon », Revue de droit comparé du travail et de la sécurité sociale, 2017/2, pp. 62-69.

66 P. Hsiao, «Power Harassment: The Tort of Workplace Bullying in Japan », op. cit., sp. p.183.

67 P. Hsiao, ibid, spéc. p. 188.

68 P. Hsiao, «Power Harassment: The Tort of Workplace Bullying in Japan », op. cit., spéc. p. 190. 
ventilateur pour faire souffler de l'air froid en hiver, et obligé les salariés à écrire des excuses et s'accuser de ne pas travailler tout en recevant un salaire. Il avait également donné des coups de pied et frappé certains salariés ${ }^{69}$.

\section{B - LA TIMIDITÉ DES JUGES ESPAGNOLS ET AMÉRICAINS}

Dans d'autres pays, le juge a fait preuve de moins d'audace ou d'inventivité. Ainsi en Espagne, on peut regretter la relative inertie du droit prétorien. Ainsi que le fait remarquer le professeur Cristóbal Molina Navarrete ${ }^{70}$, le juge a adopté une définition trop étroite de la notion de harcèlement, faisant échec à un grand nombre de demandes en réparation des salariés. Le droit constitutionnel a parfois été utilisé afin de sanctionner les attitudes managériales les plus graves, notamment au travers de la violation du droit fondamental à l'intégrité humaine. Le juge espagnol est réticent à sanctionner certains débordements qui, selon lui, relèvent du fonctionnement normal de l'entreprise. Même si le droit pénal offre des ressources, il y a peu de condamnations d'employeurs ayant harcelé ou toléré un harcèlement.

Plus extrême encore est l'exemple du droit américain ${ }^{71}$. Le Titre VII de la célébrissime loi Civil Rights Act de 1964 énumère les motifs discriminatoires prohibés par le droit fédéral72. Cette liste est complétée au niveau des Etats fédérés par d'autres motifs interdits ${ }^{73}$. La plupart des États fédérés interdisent non seulement les mesures discriminatoires fondées sur ces motifs mais également le harcèlement causé par de telles raisons. Ainsi, l'employeur ne peut rompre un contrat de travail en raison de la religion d'un salarié (ce qui serait une discrimination) et ne peut harceler un salarié pour ce motif ${ }^{74}$.

Toutefois, le harcèlement moral en soi n'est pas prohibé. La seule ressource offerte aux salariés victimes de tels comportements est de se tourner vers le droit de la responsabilité délictuelle. Plus précisément, deux délits sont envisageables : les voies de fait (battery) ou l'infliction intentionnelle de la détresse émotionnelle (intentional infliction of emotional distress). Le premier chef de demande peut être utilisé lorsque l'employeur ou un supérieur

69 P. Hsiao, ibid., sp. p. 193.

70 Voir l'article de cet auteur dans ce numéro.

71 Voir également l'article du Professeur Camille Hebert publié dans ce numéro.

72 Sont interdites les discriminations fondées sur la race, la couleur, la religion, le sexe, ou l'origine nationale. Deux autres lois fédérales doivent être prises en compte : le Age Discrimination in Employment Act de 1967 et le Americans with Disabilities Act de 1990 prohibant certaines discriminations en raison de l'âge et du handicap.

73 A titre d'exemple l'Alabama interdit également les discriminations en raison de l'âge ; L'Alaska a ajouté à cette liste les discriminations en raison du statut familial ou de la grossesse. Certains Etats ont même prohibé des motifs discriminatoires qui n'ont pas été envisagés en France. Par exemple, la Californie interdit les discriminations fondées sur le fait qu'une femme ait allaité ou non.

74 L'Equal Employment Opportunity Commission, chargée de veiller au respect du droit fédéral en matière de discrimination donne une définition du harcèlement sur son site internet. "Le harcèlement est une conduite importune qui est basée sur la race, la couleur. Le harcèlement devient illégal lorsque 1) la conduite qui offense perdure au point de devenir récurrente, ou 2) la conduite est suffisamment grave ou envahissante pour créer un environnement de travail qu'une personne raisonnable considérerait comme intimidant, hostile ou abusif ».

https://www.eeoc.gov/laws/types/harassment.cfm 
hiérarchique se livre à des violences physiques ou des attouchements inappropriés ${ }^{75}$. Le second peut être employé lorsque des conduites intolérables de l'employeur ou son représentant rendent insupportable la poursuite du contrat de travail.

Une illustration est fournie par une décision du Texas ${ }^{76}$ qui a admis la responsabilité de l'employeur en raison des menaces physiques et verbales subies par certains salariés, de la vulgarité constante du harceleur, de ses tentatives répétées d'humilier intentionnellement et d'embarrasser les salariés, et du fait qu'il criait constamment et faisait travailler les salariés dans un climat de terreur. Cependant, ce qui est considéré comme une conduite intolérable pour la mise en jeu de la responsabilité délictuelle doit être extrêmement grave. Par exemple, dans le cas précédemment mentionné, la Cour suprême du Texas avait indiqué dans un obiter dictum que le comportement d'un employeur peut être «insensible, indiscret, mesquin, officieux, autoritaire, et vindicatif " sans atteindre la dimension requise pour justifier une responsabilité délictuelle.

II en résulte que des situations qui constitueraient en France un cas de harcèlement moral ou au Japon de power harassment, n'engendreraient aucune conséquence pour le harceleur aux Etats-Unis.

Le droit américain est donc radicalement opposé au droit continental. Deux chercheurs avaient, dès 2003, noté une évolution du modèle européen en la matière ${ }^{77}$. Les Etats-Unis ont été pionniers en matière de lutte contre les discriminations, principalement sexuelles. Ce faisant la notion de harcèlement semble inséparable du motif discriminatoire prohibé. Ce modèle n'a jamais été reçu avec enthousiasme en Europe qui a prohibé les discriminations mais qui a choisi de pousser plus loin la protection des salariés. C'est ce que traduit l'interdiction du harcèlement moral : la volonté d'étendre à la communauté des travailleurs le droit de ne pas subir de persécutions sur les lieux de travail, pour quelque motif que ce soit. Ce faisant le modèle européen est passé d'une interdiction des discriminations à la préservation de la dignité des salariés. C'est cette évolution que le droit américain refuse pour l'instant en dépit des appels réitérés de la doctrine ${ }^{78}$ et de quelques décisions prétoriennes éparses.

75 Par exemple dans une décision de 2008, une juridiction du district de Columbia rejeta une demande de harcèlement sexuel d'une plaignante mais accueillit celle de voies de fait au motif que le supérieur hiérarchique avait touché à plusieurs reprises la salariée. Evans v. Wash. Ctr. for Internships \& Academic Seminars, 587 F. Supp. 2d 148, 149-51 (D.D.C. 2008).

76 GTE Southwest, Inc. v. Bruce, 998 S.W.2d 605, 613-15 (Tex. 1999).

77 G. S. Friedman, J. Q. Whitman, «The European Transformation of Harassment Law: Discrimination versus Dignity ", Columbia Journal of European Law, vol. 9, 2003, pp. 241-274.

78 A cet égard se reporter au travail du professeur David Yamada, "The Phenomenon of 'Workplace Bullying' and the Need for Status-Blind Hostile Work Environment Protection », op. cit. ; " Crafting a legislative response to workplace bullying », Employee Rights and Employment Policy Journal, 2004, Vol. 8, pp. 475-521; "Human dignity and American employment law », University of Richmond Law Review, 2009, vol. 43, pp. 523-569; "Workplace bullying and American employment law: A ten-year progress report and assessment », Comparative Labor Law \& Policy Journal, 2010, vol. 32, $n^{\circ} 1$, pp. 251-284; "Emerging American legal responses to workplace bullying », Temple Political \& Civil Rights Law Review, 2013, vol. 22, pp. 329-354. 


\section{JURISPRUDENCE SOCIALE COMPARÉE}

\section{Conclusion}

Le suicide de la japonaise Matsuri Takahashi en décembre 2015 était passé inaperçu au plan international, avant de bénéficier d'une grande notoriété un an plus tard. Cette salariée avait mis fin à ses jours en raison de l'épuisement professionnel qu'elle éprouvait. La question du Karojisatsu (suicide causé par la surcharge de travail) est proche de celle du harcèlement moral. Dans un cas comme dans l'autre le milieu de travail devient toxique pour le salarié.

Chaque pays a adopté la réponse qui lui semble la plus appropriée. Cela peut aller d'un solide encadrement légal et prétorien (France) à une quasi absence de protection (EtatsUnis). Toutefois, ce phénomène, quel que soit le vocable sous lequel on le désigne est une constante du monde du travail moderne et suscite l'attention de la communauté scientifique mondiale comme en témoigne le Congrès international de Juin 2018 sur le Harcèlement au travail ( $11^{\text {ème }}$ édition) organisé par le COMPTRASEC à Bordeaux ${ }^{79}$.

$7911^{\text {ème }}$ Congrès international sur le harcèlement au travail. Bullying 2018 : «Mieux comprendre le harcèlement au travail dans un monde en mutation ». 5-8 Juin 2018, Bordeaux.

https://bullying2018.sciencesconf.org/?forward-action=index\&forward-controller=index\&lang=fr 